\title{
Browser Design and Analysis based on Java Language
}

\author{
Mian Yi, Bin Liu and Yan Zhang \\ Jiangxi Technical College of Manufacturing, Department of Information Engineering
}

\author{
Keywords: Web Server; Browser; C/S Structure; Java
}

\begin{abstract}
With the continuous development of network technology, the informatization process is speeding up and has become a widespread concern to access to the Internet rapidly and accurately and gets the resources. Now more commonly used Web browser is IE developed by Microsoft, and it can be used to easily check the source file, clear historical record, set the security level, and set up a proxy server, etc. In this paper, based on $\mathrm{C} / \mathrm{S}$ architecture and according to the basic principle and method of software engineering, the system is implemented with Java language and Eclipse development tools. The system is divided into client and server, and the client is browser, which has the basic functions of capturing and displaying web page, viewing the source code, and storing pages on the server and other basic functions; the server provides Web services. This paper elaborates on the technology background of Web server and browser and its detailed design and implementation.
\end{abstract}

\section{Introduction}

JAVA language is a new generation of object-oriented network programming language launched by Sun Company, and it can greatly enhance the interactivity of the page by applying it on the web page, enriching page and making the page more attractive. For Internet users, it can provide more rich information content. The birth of JAVA language is an important technological invention, which push Internet application to a new peak.

According to the data "bottleneck" formed by the database server in browser/server model structure, the paper puts forward to increasing a distributed structure of browser/server/server model consisted of WWW server between the browser and database servers. The model in the application of remote fault diagnosis system is illustrated by an example, giving the system software platform structure that WWW server completes the remote acquisition, signal processing and fault diagnosis and other data processing, introducing how to use Java language to achieve the connection among clients, servers and the database systems, and its working process of remote fault diagnosis.

\section{Features of Java}

Java is just known by people due to have the following a series of characteristics below. Simplicity: this is a significant characteristic in Java. The simple programming is the feature every application developer likes. Java is very close to $\mathrm{C}++$, but it abandons a lot of functions that are difficult to use or almost no use. Java does not support the operator overloading, polymorphic inheritance and widely automatic forcing and other functions, increasing the memory space automatic collection (i.e., automatically allocate and free memory space) function.

Distribution: Java is designed for network. In order to support the TCP/IP protocol and FTP protocol in Internet, Java provides additional routines. Java applications are free to open and access objects on the network, just like the objects in the local file system.

High security: Java security mechanism is divided into several levels. Java compiler is to ensure no source code violation of security principle: Java byte code needs a validation process before running; Loader should be ensured the loaded software from the network or file system does not violate the name space and access restrictions.

Interpretation: Java is an interpretation system. The connection process is very simple, so it's easy to develop the Applet (Java applications). All kinds of information produced during the compilation is part of the byte code, and it can be used in the connection and for the late type checking, so Java application is easier to debug. 
Multithreading: Multithreaded programming is more complex, and the threads' synchronization needs to be considered carefully. Because the Java has a set of synchronization primitives, which uses surveillance and indictor rules to provide more support for the multithreaded designer.

\section{Security Architecture of Java}

Fig 1 shows the standard components of Java security architecture. In the lower part of the chart, the core of Java 2 security architecture and Java Cryptography Architecture (JCA, Java Cryptography Architecture) are shown, and these two parts constitute Java 2 security Platform attached with Java 2 Platform (Platform). The upper part is independent of the Java 2 platform, but related to standard Java security extensions of different aspects.

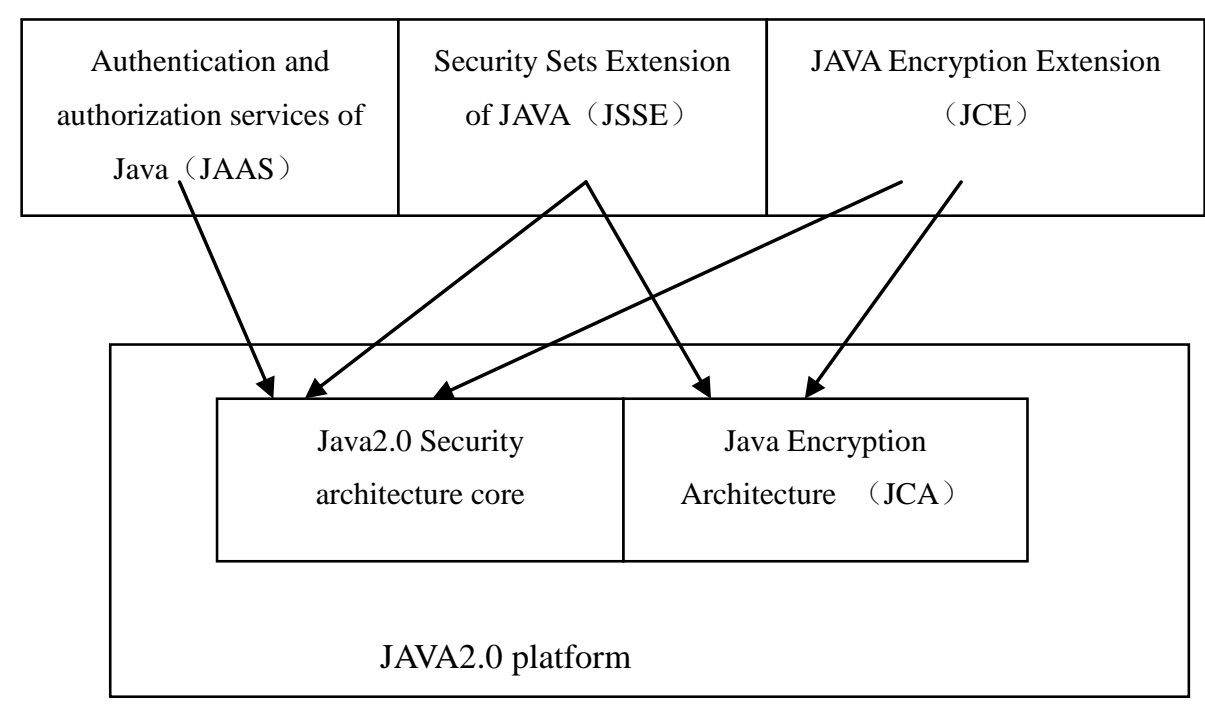

Figure 1. JAVA security standard components

The core of the Java security architecture is operated in the Java 2 platform, operating system, resources and the status of the Java code running on a Java 2 platform. The core of Java security architecture includes bytecode verifier, class loader, security manager, access controller, permissions, and strategy and protection domain.

Java cryptography architecture provides a basic cryptography tool using Java platform. Cryptography function scope includes using the basic cryptography and algorithm to protect data integrity, to prevent the destruction of data. JCA also has the algorithm generated from encrypted signature of identified data and code source.

Java Cryptography Extension (JCE, Java Cryptography Extension) is a Java auxiliary cryptography assisting security extensions. There are two English words for cryptography: cryptography and cryptography, both can be used interchangeably.

\section{Detailed Design of the System}

The interface design is shown as Fig. 2 


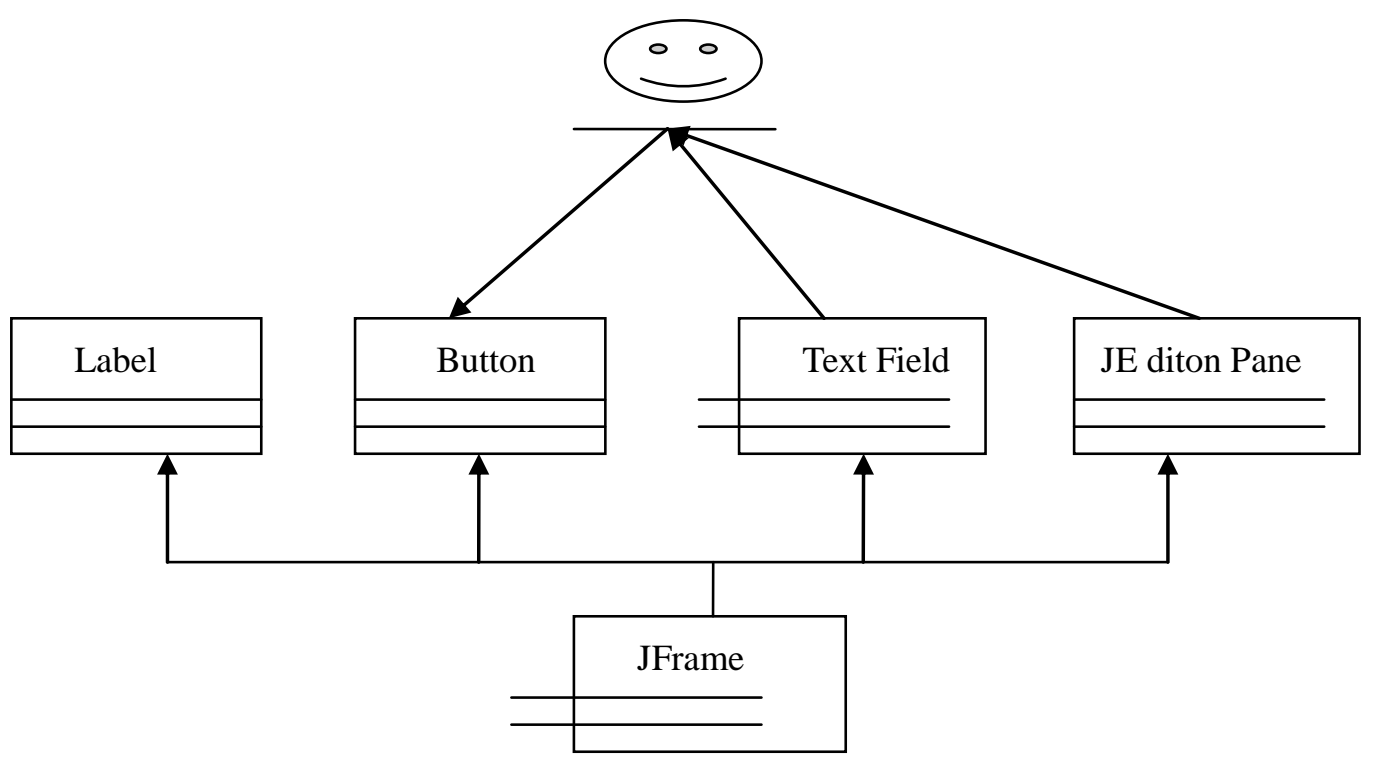

Figure 2. Interface design

\section{Function Design}

The main body framework class of Web browser is Web Browser class, which implements the events listening of each component on the framework. It mainly includes four modules: the construction of graphical user interface, the implementation of components listening interface, realization of files saving function and viewing the creation of source framework. The class diagram is shown in figure

\begin{tabular}{|l|}
\hline \multicolumn{1}{|c|}{ WebBrowse } \\
\hline +JToolBar \\
+JTextField \\
+JMenuBar \\
+JMenu \\
+JMenuItem \\
\hline +WebBrower \\
- actionPerformed (ActionEvent e):void \\
- saveFile(final String url):void \\
- getHtmlSource(String url):void \\
- hyperlink Update(HyperlinkEvent e):void \\
- main (String [] args):void \\
\hline
\end{tabular}

Figure 3. Class diagram of WebBrower

In Fig.3:

JToolBar, JTextField, JMenuBar, JMenu, JMenuItem are the attributes relative to interface. actionPerformed (ActionEvent e):void is the action-Performed function used to realize listener interface.

saveFile(final String url):void is used to save files.

getHtmlSource(String url):void is used to get the source code.

hyperlink Update(HyperlinkEvent e):void is the hyperlink Update method to realize hyperlink event listener interface.

Main(String [] Args):Void Generates an IE Object, Which is the Program Entrance. In this core class, each functional module in the general structure design will be realized respectively. The 
main function of ViewSourceFrame class is to realize the main frame of source file check and the saving function of source files.

Build Browser Connection. The realization process of building browser connection: use actionPerformed() method to respond ActionEvent event to build the connection.

Input the address in address bar and click turn to button, e.getSource ()$=$ button, url $=$ jurl.getText() to get the contents of address bar.

If url is not null and starts with http://, JEditorPane component will display the content link of url, and add the contents of url into the history of ArrayList object. Set the value of historyIndex and re-layout, call jEditorPane1.revalidate ().

If the link is shown failed, the selection dialog box "Cannot open this searching page" will pop up, then call JOption-Pane.showMessageDialog (WebBrowser.this, "Cannot open this searching page", "web browser", JOp-tionPane.ERROR_MESSAGE).

If url is not null and doesn't start with http://, url= "http://"+url, then add http:// automatically.

\section{Conclusion}

After a period of design and development, Web server and browser are finally finished and their basic functions have been implemented that the browser can access the Web server, at the same time the source code can be viewed and the page can be saved through inputting the correct URL access to get hypertext and display; the server can provide Web services. But due to the influence of various factors, the design is slightly hasty that results in the system having a lot of regrettable points, such as the user interface is not beautiful, the functions of refreshing the web page, page collection are not realized and error handling is thoughtless, which are yet to be further improved. To do well in this work, it must be under the premise to master necessary theoretical knowledge, with constant practice, accumulating experience, and improving the ability to analyze and solve problems.

\section{References}

[1] O'Brien M C, Bown C W, Merritt D E. On-line marketing optimization and design method and system: US, US 20080010142 A1[P]. 2008.

[2] Ball T J, Douglis F. Method and apparatus for tracking and viewing changes on the web: US, US 6366933 B1 [P]. 2002.

[3] Noda T. Web server, web server having function of Java servlet, and computer readable medium: US, US 8533587 B2 [P]. 2013.

[4] Orozova D A. Establishing of a Web-based Information System Using Java Instruments Practical Course [J]. Academic Open Internet Journal, 2005(14).

[5] Banavar G S, Cardone R J, Hirose S I, et al. Method, apparatus, and program for application design based on diagram specialization: US, US20050229153[P]. 2005.

[6] Berstis V, Rodriguez H. Blocking saves to web browser cache based on content rating: US, US6510458 [P]. 2003.

[7] Varga M, Lapin I, Kacur J. Performance evaluation of GMM and KD-KNN algorithms implemented in speaker identification web-application based on Java EE[C]// 2014 56th International Symposium ELMAR. 2014:1-4.

[8] Aupperle B E, Peters M L. Apparatus and method for determining compatibility of web sites with designated requirements based on functional characteristics of the web sites: US, US 7254526 B2[P]. 2007.

[9] Karidi R, Shalom D B, Zucker A, et al. System and method for design and dynamic generation of a web page: US, US20070061412[P]. 2007. 
[10]Ballard C L. Varying web page link based on user and web page status: US, US6449765 [P]. 2002.

[11]Avni Y, Suchard E. System for and method of web signature recognition system based on object map: US, US6687390 [P]. 2004.

[12]Kay E. Managing permissions and capabilities of web applications and browser extensions based on install location: US8935755 [P]. 2015. 